\title{
Impact of the stability bound choice on the approximation of ruin probabilities
}

\author{
Aicha Bareche $^{1, *}$, Mouloud Cherfaoui ${ }^{1,2}$ and Djamil Aïssani ${ }^{2}$ \\ ${ }^{1}$ Research Unit LaMOS (Modeling and Optimization of Systems), University of Bejaia, 06000 Bejaia, \\ Algeria \\ ${ }^{2}$ Department of Mathematics, University of Biskra, 07000 Biskra, Algeria
}

Received August 10, 2016; Accepted October 20, 2016

Copyright (c) 2016, Afrika Statistika and Statistics and Probability African Society (SPAS). All rights reserved

\begin{abstract}
This paper aims to establish the effect of the choice of a stability bound for the ruin probability on the quality of the approximation of the characteristics (ruin probabilities) of two classical risk models to approach (ideal an perturbed models) regarding to different large claims. In particular, we use two versions of the strong stability method: strong stability of a Markov chain and strong stability of a Lindley process. A comparative study, based on numerical results obtained by simulation, is performed between the two versions.
\end{abstract}

Résumé. Ce travail porte sur l'étude de l'effet du choix de la borne de stabilité pour la probabilité de ruine sur la qualité de l'approximation des caractéristiques (probabilités de ruine) de deux modèles de risque classiques à approcher (modèle idéal et modèle perturbé) par rapport à différentes réclamations larges. Particulièrement, on utilise deux versions de la méthode de stabilité forte: stabilité forte d'une chaîne de Markov et stabilité forte d'un processus de Lindley. Une étude comparative, basée sur des résultats numériques obtenus par simulation, est effectuée entre ces deux versions.

Key words: Risk model, perturbation, ruin probability, strong stability, large claim, simulation.

AMS 2010 Mathematics Subject Classification : Primary: 91B30 ; Secondary: 34K20, 91G70, 62G32.

${ }^{*}$ Corresponding author Aicha Bareche : aicha_bareche@yahoo.fr

Mouloud Cherfaoui : mouloudcherfaoui2013@gmail.com

Djamil Aïssani : lamos_bejaia@hotmail.com 
A. Bareche, M. Cherfaoui and D. Aïssani, Afrika Statistika, Vol. 11(2), 2016, pages 983-993. Impact of the stability bound choice on the approximation of ruin probabilities.

\section{Introduction}

In the last decades, the specialists became aware of the importance of the stability analysis in actuarial sciences and financial mathematics. The ruin theory has the objective of modeling the evolution of the wealth of an insurance company by a stochastic process and evaluating its ruin probability. This measure is one of the basic characteristics of risk models and various authors investigated the problem of its evaluation (see Asmussen, 2000; Panjer and Willmot, 1992). However, it cannot be expressed in an explicit form for many risk models. Furthermore, the parameters governing these models are often unknown and one can only give some bounds for their values. In such a situation the question of stability becomes crucial. There resides the interest of obtaining explicit stability bounds for ruin probabilities of different risk models.

In the stability theory, we establish the domain within which a model may be used as a good approximation or idealization of the real system under consideration. In other words, here, we clarify the conditions for which the proximity in one way or another of the parameters of the system involve the proximity of the studied characteristics. Such results give the possibility of approximating some complicated systems by other systems more exploitable and much simpler (See Beirlant and Rachev, 1987, Kalashnikov and Tsitsiashvili, 1973, Kartashov, 1986, Kartashov, 1996).

Many authors looked at the problem of stability of actuarial models. Beirlant and Rachev (1987) introduced the problem of stability in insurance mathematics. Kalashnikov (2000) investigated the estimation of ruin probabilities in the univariate risk models, using the strong stability method, the reversed process notion and the supplementary variables technique. Then, other authors (see Aïssani and Benouaret, 2010, Enikeeva et al., 2001) extended the application of this approach to different types of risk models.

In practice, finding a good-fitting distribution for large data sets that contain some relatively large claim amounts, such as insurance claims, is not an easy task. It is worthy to notice the special importance of heavy-tailed distributions, which is more and more used in the last years because of occasional appearance of huge claims (See Coles, 2001, Embrechts et al., 1997, Embrechts and Veraverbeke, 1982, Konstantinidis, 2007, Tsitsiashvili and Konstantinides, 2001). Indeed, It is important to use an appropriate distribution to well fit the bulk of the data and capture adequately atypical extreme observations. But, often actuaries use standard models such as the lognormal, Weibull and Pareto distributions or a mixture of lognormal and Pareto distributions. It is why we choose, in the context of this work, these most popular specifications.

The aim of this work is to provide a new stability bound for the ruin probability in a classical risk model, using the the concept of the strong stability of a Lindley process. For a comparison purpose and in order to test the performance of this approach, we contrast this latter to a closer one, for instance a stability bound based on the strong stability of a Markov chain Kalashnikov (2000). We perform several simulations, involving different large claims distributions, in order to study the influence of the chosen stability bound on the quality of the approximation of ruin probabilities of two risk models to approach (ideal and perturbed models).

Journal home page: www.jafristat.net ; www.projecteuclid.org/as 
A. Bareche, M. Cherfaoui and D. Aïssani, Afrika Statistika, Vol. 11(2), 2016, pages 983-993. Impact of the stability bound choice on the approximation of ruin probabilities.

\section{Description of a univariate classical risk model}

In the actuarial literature, the evolution in time of the capital of an insurance company is often modeled by the process of reserve resulting from the difference between the premiumincome and the pay-out process. The classical risk process in the one-dimensional situation can be stated as

$$
X(t)=u+c t-Z(t), \quad t \geq 0,
$$

where $X(t)$ is the surplus of an insurance company at time $t \geq 0, u \geq 0$ the initial surplus, $c$ the rate at which the premiums are received, and $Z(t)$ the aggregate of the claims between time 0 and $t . Z(t)=\sum_{i=1}^{N(t)} Z_{i}$, where $\left\{Z_{i}, i \geq 1\right\}$ is a sequence of iid random variables, representing the claim amounts of distribution function denoted by $F(x)$ and mean claim size denoted by $\mu,\{N(t), t \geq 0\}$ being a Poisson process with parameter $\lambda$, representing the number of claims. The relative security loading $\theta$ is defined by $\theta=\frac{c-\lambda \mu}{\lambda \mu}$. We further assume that $c>\lambda \mu$, the expected payment per unit of time.

Ruin theory for the univariate risk process defined in Formula (1) has been extensively discussed in the literature (Asmussen, 2000, Panjer and Willmot, 1992).

\section{Strong stability of a univariate classical risk model}

\subsection{Strong stability criterion}

For a general framework on the strong stability method, the reader is referred to Kartashov (1986) and to Kartashov (1996). However, let us recall the following basic definition.

Definition 1. (Kartashov, 1996) A Markov chain $X$ with transition kernel $P$ and invariant measure $\pi$ is said to be $v$-strongly stable with respect to the norm ( $\|\alpha\|_{v}=$ $\int_{0}^{\infty} v(x)|\alpha|(d x)$, for a measure $\left.\alpha\right)$, if $\|P\|_{v}<\infty$ and each stochastic kernel $Q$ in some neighborhood $\left\{Q:\|Q-P\|_{v}<\epsilon\right\}$ has a unique invariant measure $\mu=\mu(Q)$ and $\|\pi-\mu\|_{v} \rightarrow 0$ as $\|Q-P\|_{v} \rightarrow 0$.

The general aspect of the concept of stability in risk models can be stated as follows: Suppose that the governing parameter $a$ of a risk model takes its values in the metric space $(\mathbb{A}, \mu)$. For a fixed parameter $a$, we consider the ruin probability $\Psi_{a}(x)$ as a function of the initial capital $x$ and assume that the space $\Psi=\left\{\Psi_{a}(x), a \in \mathbb{A}\right\}$ is equipped with a metric $\nu$. The ruin probability $\Psi_{a}(x)$ is said to be stable at point $a$ if for any sequence $\left\{a^{\prime}\right\} \subset \mathbb{A}$ which converges to $a$, we have the convergence of the corresponding ruin probabilities, i.e.

$$
\left(\mu\left(a, a^{\prime}\right) \rightarrow 0\right) \Rightarrow\left(\nu\left(\Psi_{a}, \Psi_{a^{\prime}}\right) \rightarrow 0\right) .
$$

Moreover, if one can find a continuous function $f$ in $0(f(0)=0)$ and a subset $\mathbb{A}_{d} \subset \mathbb{A}$ such that:

$$
\nu\left(\Psi_{a}, \Psi_{a^{\prime}}\right) \leq f\left(\mu\left(a, a^{\prime}\right)\right), \quad a, a^{\prime} \in \mathbb{A}_{d},
$$

we call it (this latter equation) stability bound of the ruin probability. In general, the function $f$ depends on the parameters $a, a^{\prime}$.

Let us denote the reversed process associated to the risk model defined in (1) by $\left\{V_{n}\right\}_{n \geq 0}$. The strong stability approach consists in identifying the ruin probability $\Psi_{a}(u)$ associated to

Journal home page: www.jafristat.net ; www.projecteuclid.org/as 
the risk model governed by a vector parameter $a=(\lambda, \mu, c)$, with the stationary distribution of the reversed process $\left\{V_{n}\right\}_{n \geq 0}$, i.e.

$$
\Psi_{a}(u)=\lim _{n \rightarrow \infty} \mathbb{P}\left(V_{n}>u\right)
$$

where $u$ is the initial reserve. (See Kalashnikov, 2000).

\subsection{Stability inequalities}

Let $a^{\prime}=\left(\lambda^{\prime}, \mu^{\prime}, c^{\prime}\right)$ be the vector parameter governing another univariate risk model defined as above. Its ruin probability and its reversed process are denoted $\Psi_{a^{\prime}}(u)$ and $\left\{V_{n}^{\prime}\right\}_{n \geq 0}$, respectively.

\subsubsection{Version 1: Stability of a Markov chain}

The following theorem determines the $v$-strong stability conditions of a univariate classical risk model using the general theory of Markov chains. It also gives the estimates of the deviations between both transition operators and both ruin probabilities in the steady state.

Theorem 1. (Kalashnikov, 2000) Consider a univariate classical risk model governed by a vector parameter $a$. Then, there exists $\varepsilon>0$ such that the reversed process $\left\{V_{n}\right\}_{n \geq 0}$ (Markov chain), which is associated to this model, is strongly stable with respect to the weight function $v(x)=e^{\epsilon x} \quad(\epsilon>0), \quad x \in \mathbb{R}^{+}$.

In addition, if

$$
\mu\left(a, a^{\prime}\right)<(1-\rho(\epsilon))^{2}
$$

then we obtain the margin between the transition operators $P$ and $P^{\prime}$ of the Markov chains $\left\{V_{n}\right\}_{n \geq 0}$ and $\left\{V_{n}^{\prime}\right\}_{n \geq 0}$ :

$$
\left\|P-P^{\prime}\right\|_{v} \leq 2 \mathbb{E} e^{\epsilon Z}\left|\ln \frac{\lambda c^{\prime}}{\lambda^{\prime} c}\right|+\left\|F-F^{\prime}\right\|_{v}
$$

where,

$$
\begin{aligned}
\mu\left(a, a^{\prime}\right) & =2 \mathbb{E} e^{\epsilon Z}\left|\ln \frac{\lambda c^{\prime}}{\lambda^{\prime} c}\right|+\left\|F-F^{\prime}\right\|_{v} \\
\rho(\epsilon) & =\mathbb{E}\left(\exp \left\{\epsilon\left(Z_{1}-c U\right)\right\}\right), \\
\left\|F-F^{\prime}\right\|_{v} & =\int_{0}^{\infty} v(u)\left|d\left(F-F^{\prime}\right)\right|(u)=\int_{0}^{\infty} v(u)\left|f-f^{\prime}\right|(u) d u .
\end{aligned}
$$

Moreover, we have the deviation between the ruin probabilities:

$$
\left\|\Psi_{a}-\Psi_{a^{\prime}}\right\|_{v} \leq \frac{\mu\left(a, a^{\prime}\right)}{(1-\rho(\epsilon))\left((1-\rho(\epsilon))^{2}-\mu\left(a, a^{\prime}\right)\right)}=\Gamma_{1} .
$$

Journal home page: www.jafristat.net ; www.projecteuclid.org/as 


\subsubsection{Version 2: Stability of a Lindley process}

In the case of a collective risk model (See Kalashnikov, 2000), the dual process (reversed process) of the reserve process $X(t)$ defined in (1) can be simply written, for all $n \geq 0$ as follows:

$$
V_{n+1}=\left(V_{n}-c U+Z_{n+1}\right)_{+}, \quad V_{0}=0,
$$

where $U$ is a random variable following an exponential distribution with parameter $\lambda$.

The process $V=\left\{V_{n}, n \geq 0\right\}$ is a Lindley process (see Kalashnikov, 1978, Kalashnikov, 2000; Kalashnikov and Tsitsiashvili, 1973), with a governing sequence $\left\{\xi_{n}, n \geq 0\right\}$ such that $\xi_{n+1}=Z_{n+1}-c U$, and it is defined on the phase space $\left(\mathbb{R}_{+}, \mathcal{B}\left(\mathbb{R}_{+}\right)\right)$, with $\mathcal{B}\left(\mathbb{R}_{+}\right)$is the Borel $\sigma$-algebra on $\mathbb{R}_{+}$. Consequently, based on the properties of dual processes (See Enikeeva et al., 2001, Aïssani and Benouaret, 2010), it is possible to write the ruin probabilities in finite time $\Psi_{n}(x)$ and in infinite time $\Psi(x)$ as follows:

$$
\begin{gathered}
\Psi_{n}(x)=\mathbb{P}\left(V_{n}>x\right), \quad n \geq 0, \\
\Psi(x)=\lim _{n \rightarrow+\infty} \Psi_{n}(x)=\lim _{n \rightarrow+\infty} \mathbb{P}\left(V_{n}>x\right) .
\end{gathered}
$$

Thus, from relations (5) and (6), the basic characteristic of the model can be expressed by the set of the following cumulative functions:

$$
\begin{gathered}
\mathbb{G}_{n}(x)=1-\mathbb{P}\left(V_{n}>x\right), \quad n \geq 0, \\
\mathbb{G}(x)=\lim _{n \rightarrow+\infty} \mathbb{G}_{n}(x)=1-\lim _{n \rightarrow+\infty} \mathbb{P}\left(V_{n}>x\right) .
\end{gathered}
$$

Besides, according to relation (6), the ruin probability is linked to the stationary distribution of the dual process $V=\left\{V_{n}, n \geq 0\right\}$ which forms an homogeneous Markov chain.

To be able to prove the last theorem of the subsequent section (Theorem 4), we state the two following intermediate results (Theorem 2 and Theorem 3):

Theorem 2. (Kartashov, 1986) Suppose that $\mathbb{E}(\xi)<0$ and there exists $\theta>0$ such that $\mathbb{E}[\exp (\theta \xi)]<\infty$. Then, the Markov chain $V$ is aperiodic and uniformly ergodic according to $\|\cdot\|_{v}$ for all $\epsilon$ such that $\rho(\epsilon)=\mathbb{E}[\exp (\epsilon \xi)]<1$.

Theorem 3. Suppose that the conditions of Theorem 2 are fulfilled. Then, for all $\epsilon$ such that $\rho(\epsilon)=\mathbb{E}[\exp (\epsilon \xi)]<1$ and $\gamma(\epsilon)=\left\|P-P^{\prime}\right\|_{v}<1-\rho(\epsilon)$, we have the following inequality:

$$
\left\|\pi-\pi^{\prime}\right\|_{v} \leq \frac{1}{(1-\rho(\epsilon)-\gamma(\epsilon))^{2}}\left(\frac{2-\rho(\epsilon)}{1-\rho(\epsilon)}\right)\left\|P-P^{\prime}\right\|_{v}
$$

The proof of Theorem 3 is based on the results of Theorem 3.5 given in Kartashov (1996).

Now, the following theorem gives new quantitative estimates for the ruin probability of the model defined in (1) based on the strong stability of a Lindley process Kalashnikov (2000).

Theorem 4. Let $\epsilon>0$ such that $\rho(\epsilon)=\mathbb{E}[\exp (\epsilon(Z-c U))]<1$. If

$$
\mu\left(a, a^{\prime}\right)<1-\rho(\epsilon)
$$

Journal home page: www.jafristat.net ; www.projecteuclid.org/as 
then we obtain the margin between the ruin probabilities of the Markov chains $\left\{V_{n}\right\}_{n \geq 0}$ and $\left\{V_{n}^{\prime}\right\}_{n \geq 0}$ :

$$
\left\|\Psi_{a}-\Psi_{a^{\prime}}\right\|_{v} \leq \frac{\mu\left(a, a^{\prime}\right)(2-\rho(\epsilon))}{(1-\rho(\epsilon))\left(1-\rho(\epsilon)-\mu\left(a, a^{\prime}\right)\right)^{2}}=\Gamma_{2} .
$$

Proof. Consider the two stationary distributions $\pi=\left(\pi(\Gamma), \Gamma \in \mathcal{B}\left(\mathbb{R}_{+}\right)\right)$and $\pi^{\prime}=\left(\pi^{\prime}(\Gamma), \Gamma \in\right.$ $\mathcal{B}\left(\mathbb{R}_{+}\right)$) of the Markov chains $V$ and $V^{\prime}$, respectively. Thus, for all $x \in \mathbb{R}_{+}$, we have:

$$
\begin{aligned}
\pi([0, x]) & =\mathbb{P}\left(V_{\infty} \leq x\right) \\
& =1-\mathbb{P}\left(V_{\infty}>x\right) \\
& =1-\lim _{n \rightarrow+\infty} \mathbb{P}\left(V_{n}>x\right) \\
& =1-\Psi_{a}(x),
\end{aligned}
$$

and similarly $\pi^{\prime}([0, x])=1-\Psi_{a}^{\prime}(x)$. Therefore, we find that $\left\|\pi-\pi^{\prime}\right\|_{v}=\left\|\Psi_{a}-\Psi_{a^{\prime}}\right\|_{v}$. Consequently, by substituting estimate (3) in inequality (7), the result is immediately obtained.

Remark 1. Without loss of generality, we relax some conditions by taking $\lambda^{\prime}=\lambda, c^{\prime}=c$. Then, we have: $\mu\left(a, a^{\prime}\right)=\left\|F-F^{\prime}\right\|_{v}=\int_{0}^{\infty} v(u)\left|f-f^{\prime}\right|(u) d u$. The perturbation may concern the claim mean size (i.e. $\mu^{\prime}=\mu+\varepsilon$ ) or the claim distribution $F$ itself.

Remark 2. It is not easy to compare, in general, the quality of the two estimates (4) and (9). However, it is clear that the validity domain of estimate (9) defined by (8) is much larger than that given by (2) and linked to (4). It is why we amount, in the following section, to perform such a comparison numerically.

\section{Numerical example - Simulation}

Algoritm 1 allows us to compute the different theoretical quantities in the case of Theorem 1. In a similar way, it may also be re-used to compute the different theoretical quantities in the case of Theorem 4, by adopting the appropriate changes.

Different simulated claim distributions (with their respective parameters) are given in Table 1. Stability intervals, with the different distributions, relative to Theorem 1 and Theorem 4 are respectively summarized in Table 2 and Table 3 . Similarly, the stability bounds $\Gamma_{1}$ and $\Gamma_{2}$ are provided respectively in Table 4 and Table 5 . Figure 1 shows the variation of the stability bound $\Gamma_{1}$ (at the top), (respectively, the variation of the stability bound $\Gamma_{2}$ (at the bottom)) in function of the perturbation $\varepsilon$, with the different distributions.

Journal home page: www.jafristat.net ; www.projecteuclid.org/as 
Algorithm 1 (Computation of the stability bound $\Gamma_{1}$ for the ruin probability)

1) Introduce the parameters $\lambda, \mu, c$ of the ideal model, and $\lambda^{\prime}, \mu^{\prime}, c^{\prime}$ of the perturbed (real) model.

2) Verify the positivity of the relative security loadings $\theta$ and $\theta^{\prime}$ defined by: $\theta=\frac{c-\lambda \mu}{\lambda \mu}$ and $\theta^{\prime}=\frac{c^{\prime}-\lambda^{\prime} \mu^{\prime}}{\lambda^{\prime} \mu^{\prime}}$.

If yes, $(*$ the ruin is not sure*) go to step 3 ;

else return to step 1 .

3) Initialize $\epsilon(\epsilon>0)$ such that $0<\rho(\epsilon)<1$ and $\Gamma_{1}$ to be minimal.

4) Compute $\mu\left(a, a^{\prime}\right)=\int_{0}^{\infty} v(u)\left|f-f^{\prime}\right|(u) d u$, and test:

$\mu\left(a, a^{\prime}\right)<(1-\rho(\epsilon))^{2}$.

If yes, $\left({ }^{*}\right.$ we can deduce the strong stability inequality*) go to step 5;

else increment $\epsilon$ with step $p$, then return to step 4 .

5) Compute the bound $\Gamma_{1}$ on the deviation $\left\|\Psi_{a}-\Psi_{a^{\prime}}\right\|_{v}$ such that:

$$
\left\|\Psi_{a}-\Psi_{a^{\prime}}\right\|_{v} \leq \frac{\mu\left(a, a^{\prime}\right)}{(1-\rho(\epsilon))\left((1-\rho(\epsilon))^{2}-\mu\left(a, a^{\prime}\right)\right)}=\Gamma_{1} .
$$

\begin{tabular}{|c|c|c|c|c|}
\hline Mean & $\operatorname{Exp}(\mu)$ & Lognormal $(\alpha, \beta)$ & Weibull $(\alpha, \beta)$ & Logistic $(\mu, \sigma)$ \\
\hline 2.00 & 2.00 & $(0.5816,0.4724)$ & $(2.2397,3)$ & $(1.0000,0.7213)$ \\
\hline 2.10 & 2.10 & $(0.6398,0.4521)$ & $(2.3517,3)$ & $(1.1000,0.7213)$ \\
\hline 2.20 & 2.20 & $(0.6945,0.4334)$ & $(2.4637,3)$ & $(1.2000,0.7213)$ \\
\hline 2.30 & 2.30 & $(0.7463,0.4161)$ & $(2.5756,3)$ & $(1.3000,0.7213)$ \\
\hline 2.40 & 2.40 & $(0.7954,0.4001)$ & $(2.6876,3)$ & $(1.4000,0.7213)$ \\
\hline 2.50 & 2.50 & $(0.8421,0.3853)$ & $(2.7996,3)$ & $(1.5000,0.7213)$ \\
\hline 2.60 & 2.60 & $(0.8865,0.3714)$ & $(2.9116,3)$ & $(1.6000,0.7213)$ \\
\hline 2.70 & 2.70 & $(0.9290,0.3585)$ & $(3.0236,3)$ & $(1.7000,0.7213)$ \\
\hline 2.80 & 2.80 & $(0.9696,0.3465)$ & $(3.1356,3)$ & $(1.8000,0.7213)$ \\
\hline 2.90 & 2.90 & $(1.0085,0.3352)$ & $(3.2476,3)$ & $(1.9000,0.7213)$ \\
\hline 3.00 & 3.00 & $(1.0459,0.3246)$ & $(3.3595,3)$ & $(2.0000,0.7213)$ \\
\hline
\end{tabular}

Table 1: Different simulated distributions

\begin{tabular}{|c|c|c|c|c|c|}
\hline$\varepsilon$ & Mean & Exp & Lognormal & Weibull & Logistic \\
\hline-0.5 & 2.00 & {$[0.0002,0.3083]$} & ] $0,0.2955]$ & {$[0.0005,0.2685]$} & {$[0.0003,0.1933]$} \\
-0.4 & 2.10 & {$[0.0002,0.3320]$} & ] $0,0.3726]$ & {$[0.0004,0.3424]$} & {$[0.0002,0.2807]$} \\
-0.3 & 2.20 & {$[0.0001,0.3610]$} & ] $0,0.4645]$ & {$[0.0003,0.431]$} & {$[0.0002,0.3873]$} \\
-0.2 & 2.30 & {$[0.0001,0.3999]$} & ] $0,0.5826]$ & {$[0.0003,0.547]$} & {$[0.0002,0.5264]$} \\
-0.1 & 2.40 & {$[0.0001,0.4627]$} & ] $0,0.7565]$ & {$[0.0002,0.7306]$} & {$[0.0001,0.7357]$} \\
0.00 & 2.50 & ] $0, \infty[$ & ] $0, \infty[$ & ] $0, \infty[$ & {$[0, \infty[$} \\
+0.1 & 2.60 & {$[0.0001,0.6172]$} & $0,0.7571]$ & {$[0.0002,0.7121]$} & {$[0.0001,0.7166]$} \\
+0.2 & 2.70 & {$[0.0001,0.5295]$} & $0,0.5590]$ & {$[0.0002,0.5261]$} & {$[0.0002,0.4921]$} \\
+0.3 & 2.80 & {$[0.0001,0.4772]$} & $0,0.4330]$ & {$[0.0003,0.4145]$} & {$[0.0002,0.3465]$} \\
+0.4 & 2.90 & {$[0.0002,0.4398]$} & ] $0,0.3399]$ & {$[0.0004,0.3352]$} & {$[0.0002,0.2397]$} \\
+0.5 & 3.00 & {$[0.0002,0.4108]$} & ] $0,0.2663]$ & {$[0.0004,0.2744]$} & {$[0.0003,0.1573]$} \\
\hline
\end{tabular}

Table 2: Stability intervals according to Theorem ${ }^{1}$ 
A. Bareche, M. Cherfaoui and D. Aïssani, Afrika Statistika, Vol. 11(2), 2016, pages 983-993.

\begin{tabular}{|c|c|c|c|c|c|}
\hline$\varepsilon$ & Mean & Exp & Lognormal & Weibull & Logistic \\
\hline-0.5 & 2.00 & {$[0.0001,0.3920]$} & {$[0.0001,0.2963]$} & {$[0.0003,0.2686]$} & {$[0.0002,0.1934]$} \\
-0.4 & 2.10 & {$[0.0001,0.4265]$} & {$[0.0001,0.3739]$} & {$[0.0002,0.3425]$} & {$[0.0002,0.2808]$} \\
-0.3 & 2.20 & {$[0.0001,0.4687]$} & {$[0.0001,0.4667]$} & {$[0.0002,0.4311]$} & {$[0.0001,0.3875]$} \\
-0.2 & 2.30 & {$[0.0001,0.5250]$} & {$[0.0001,0.5875]$} & {$[0.0001,0.5471]$} & {$[0.0001,0.5269]$} \\
-0.1 & 2.40 & {$[0.0001,0.6158]$} & {$[0.0001,0.7734]$} & {$[0.0001,0.7307]$} & {$[0.0001,0.7371]$} \\
0.00 & 2.50 & {$[0.0000, \infty]$} & {$[0.0000, \infty]$} & {$[0.0000, \infty]$} & {$[0.0000, \infty]$} \\
+0.1 & 2.60 & {$[0.0001,0.6173]$} & {$[0.0001,0.7572]$} & {$[0.0001,0.7122]$} & {$[0.0001,0.7167]$} \\
+0.2 & 2.70 & {$[0.0001,0.5296]$} & {$[0.0001,0.5591]$} & {$[0.0001,0.5262]$} & {$[0.0001,0.4922]$} \\
+0.3 & 2.80 & {$[0.0001,0.4773]$} & {$[0.0001,0.4331]$} & {$[0.0002,0.4146]$} & {$[0.0001,0.3466]$} \\
+0.4 & 2.90 & {$[0.0001,0.4399]$} & {$[0.0001,0.3400]$} & {$[0.0002,0.3353]$} & {$[0.0002,0.2401]$} \\
+0.5 & 3.00 & {$[0.0001,0.4109]$} & {$[0.0001,0.2664]$} & {$[0.0002,0.2745]$} & {$[0.0002,0.1574]$} \\
\hline
\end{tabular}

Table 3: Stability intervals according to Theorem 4

\begin{tabular}{|c|c|c|c|c|c|}
\hline$\varepsilon$ & Mean & Exp & Lognormal & Weibull & Logistic \\
\hline-0.5 & 2.00 & 0.1954 & 1.0098 & 0.9509 & 2.0178 \\
-0.4 & 2.10 & 0.1463 & 0.6713 & 0.6224 & 1.1851 \\
-0.3 & 2.20 & 0.1032 & 0.4302 & 0.3943 & 0.6986 \\
-0.2 & 2.30 & 0.0649 & 0.2498 & 0.2273 & 0.3819 \\
-0.1 & 2.40 & 0.0307 & 0.1105 & 0.0999 & 0.1612 \\
0.00 & 2.50 & 0 & 0 & 0 & 0 \\
+0.1 & 2.60 & 0.0267 & 0.1087 & 0.0957 & 0.1613 \\
+0.2 & 2.70 & 0.0534 & 0.2418 & 0.2064 & 0.3819 \\
+0.3 & 2.80 & 0.0801 & 0.4068 & 0.3357 & 0.6990 \\
+0.4 & 2.90 & 0.1067 & 0.6166 & 0.4883 & 1.1861 \\
+0.5 & 3.00 & 0.1333 & 0.8915 & 0.6704 & 2.0216 \\
\hline
\end{tabular}

Table 4: Stability bound $\Gamma_{1}$ with the different distributions 
A. Bareche, M. Cherfaoui and D. Aïssani, Afrika Statistika, Vol. 11(2), 2016, pages 983-993. Impact of the stability bound choice on the approximation of ruin probabilities.

\begin{tabular}{|c|c|c|c|c|c|}
\hline$\varepsilon$ & Mean & Exp & Lognormal & Weibull & Logistic \\
\hline-0.5 & 2.00 & 0.4283 & 4.0561 & 3.7055 & 12.1606 \\
-0.4 & 2.10 & 0.3077 & 2.2427 & 2.0178 & 5.1749 \\
-0.3 & 2.20 & 0.2087 & 1.2302 & 1.0988 & 2.3718 \\
-0.2 & 2.30 & 0.1267 & 0.6243 & 0.5576 & 1.0552 \\
-0.1 & 2.40 & 0.0580 & 0.2453 & 0.2198 & 0.3769 \\
0.00 & 2.50 & 0.0000 & 0.0000 & 0.0000 & 0.0000 \\
+0.1 & 2.60 & 0.0549 & 0.2411 & 0.2096 & 0.3745 \\
+0.2 & 2.70 & 0.1126 & 0.6004 & 0.4979 & 1.0557 \\
+0.3 & 2.80 & 0.1730 & 1.1444 & 0.8963 & 2.3739 \\
+0.4 & 2.90 & 0.2362 & 1.9932 & 1.4521 & 5.1642 \\
+0.5 & 3.00 & 0.3020 & 3.3712 & 2.2370 & 12.1967 \\
\hline
\end{tabular}

Table 5: Stability bound $\Gamma_{2}$ with the different distributions
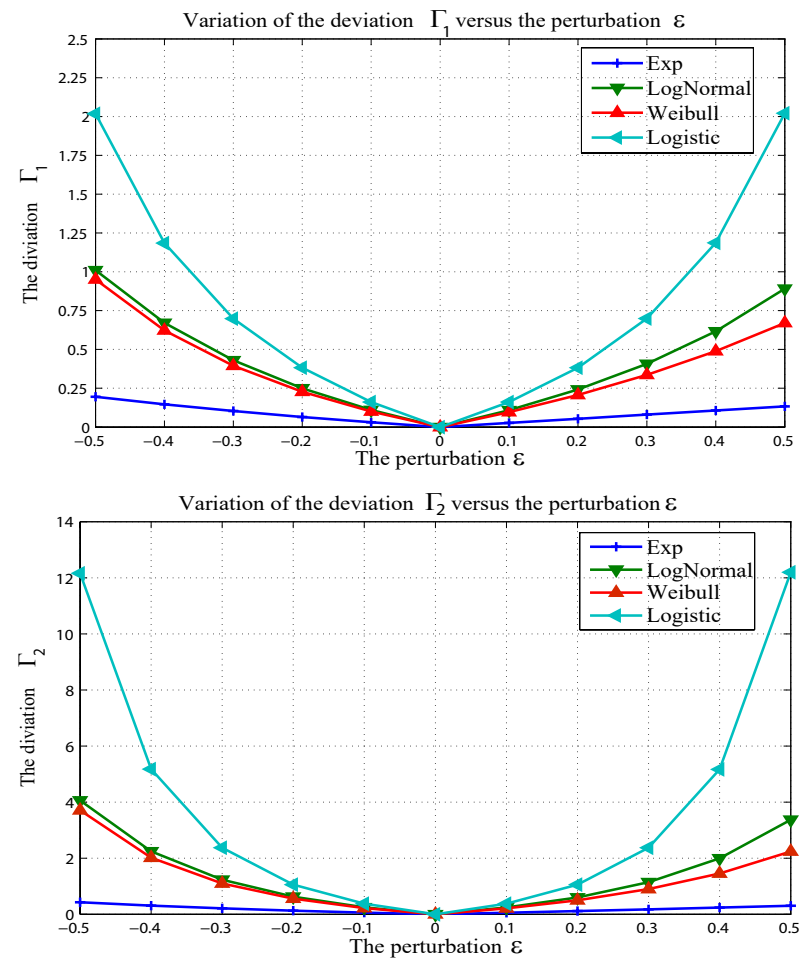

Fig. 1. Variation of the stability bound with the different distributions $\left(\Gamma_{1}\right.$ at the top and $\Gamma_{2}$ at the bottom).

Journal home page: www.jafristat.net ; www.projecteuclid.org/as 
A. Bareche, M. Cherfaoui and D. Aïssani, Afrika Statistika, Vol. 11(2), 2016, pages 983-993. Impact of the stability bound choice on the approximation of ruin probabilities.

Discussion: Based on the obtained results in Figure 1 and Tables $2-5$, we notice that: In both stability versions and for all the distributions, the stability intervals decrease with the increase of the perturbation $\varepsilon$. It is obvious that the risk model tends to be unstable with a great perturbation. Except in the case of the logistic distribution, where the stability interval is the same for both theorems, Theorem 4 provides larger stability intervals than those given by Theorem 1 (as mentioned in Remark 2). That means that we have more flexibility for the choice of the norm $v(\epsilon)$ in the second theorem (Theorem 4) than in the former (Theorem 1). In other words, the conditions of Theorem 1 are more strict than those of Theorem 4 .

The stability bounds $\Gamma_{1}$ and $\Gamma_{2}$ increase with the increase of the perturbation $\varepsilon$. Even with distributions having the same mean as that of the exponential one, one obtains sometimes bounds relatively far away from that obtained with the exponential distribution. It may be explained by the influence of the tail weight of the different considered distributions. In both stability versions, the obtained bounds for the Weibull distribution are too close to those obtained for the exponential one. It is to say, one may justify in certain situations the approximation of a risk model with a large claims distribution by another risk model governed by an exponential distribution. The behavior of the stability bound $\Gamma_{2}$ is similar to that of the bound $\Gamma_{1}$. However, the minimal error $\Gamma_{2}$ provided by the second theorem (Theorem 4) is greater than the minimal error $\Gamma_{1}$ given by the first theorem (Theorem 1 ).

\section{Conclusion and future work}

The simulation study carried out in this work shows that the obtained results of both stability versions converge in some sense, with a small preference for the stability bound of a Markov chain versus the stability bound of a Lindley process. It also shows that the approximation of the ruin probability is in connection with several criteria such as: the perturbation of the mean characteristics of the claim distribution, the choice of the norm $v(\epsilon)$ and the shape of the distribution.

In a further work, we may be interested in the approximation of the ruin probability using the strong stability method, in the case of an unknown claim distribution. Due to the particularity of this type of distributions (also known as loss distributions) which are defined on a positive bounded support, we propose to apply a semi-parametric technique that consists to estimate the underlying distribution in two steps. First, a start parametric distribution (generalized Champernowne distribution Buch-Larsen et al., 2005) can be used for estimating the initial data. Then, we shall apply the asymmetric Beta kernel estimate (See Chen, 1999) to the sample resulting from the first step. This estimator is more appropriate to this type of data because it allows the correction of the boundary effects. To avoid the problem of the non-consistency of this estimator, we may use its two normalized versions: local (mico-Beta) and global (macro-Beta) estimates (see Gouriéroux and Montfort, 2006).

\section{References}

Asmussen, S., 2000. Ruin Probabilities. World Scientific, Singapore.

Beirlant, J., Rachev, S.T., 1987. The problems of stability in insurance mathematics. Insurance Math. Econom., 6, 179-188.

Journal home page: www.jafristat.net; www.projecteuclid.org/as 
A. Bareche, M. Cherfaoui and D. Aïssani, Afrika Statistika, Vol. 11(2), 2016, pages $983-993$.

Aïssani, D. and Benouaret, Z., 2010. Modèles de Risque et Files d'Attente: La méthode de stabilité forte. Journal Afrika Statistika, 5(3), 210-218.

Buch-Larsen, T., Nielsen, J.P., Guillen, M. and Bolancé, C., 2005. Kernel density estimation for heavy-tailed distribution using the Champernowne transformation. Statistics, 6, 503518.

Chen, S.X., 1999. Beta kernel estimators for density functions. Computational Statistics and Data Analysis, 31, 131-145.

Coles, S., 2001. An Introduction to Statistical Modelling of Extreme Values. Springer, Berlin.

Embrechts, P., Klueppelberg, C., Mikosch, T., 1997. Modelling Extremal Events for Finance and Insurance. Springer-Verlag, Heildelberg.

Embrechts, P. and Veraverbeke, N., 1982. Estimates for the probability of ruin with special emphasis on the possibility of large claims. Insurance: Math. Econom., 1, 55-72.

Enikeeva, F., Kalashnikov, V., Rusaityte D., 2001. Continuity estimates for ruin probabilities, Scand. Actuar. J., 1, 18-39.

Gouriéroux, C. and Montfort, A., 2006. (Non) Consistency of the Beta Kernel Estimator for Recovery Rate Distribution. CREST-DP 2006-32.

Kalashnikov, V.V., 1978. Qualitative Analysis of Complex Systems Behaviour by Test Functions Method. Nauka, Moscow (in Russian).

Kalashnikov, V.V., 2000. The stability concept for stochastic risk models. Working Paper No. 166, Laboratory of Actuarial Mathematics, University of Copenhagen.

Kalashnikov, V.V. and Tsitsiashvili, G.Sh., 1973. On the stability of queueing systems with respect to disturbances of their distribution functions. Engineering Cybernitics, 10, 211217.

Kartashov, N.V., 1986. Strongly Stable Markov chains, Stability Problems for Stochastic Models (1981a), "Vsesoyus. Nauchno-Issled. Inst. Sistem. Issled.", Moscow, 54-59; English transl. in J. Soviet. Mat., 34, 1493-1498.

Kartashov, N.V., 1996. Strong Stable Markov chains. TbiMC Scientific Publishers, VSPV, Utrecht.

Konstantinidis, D.G., 2007. Risk models with extremal subexponentiality. Brazilian Journal of Probability and Statistics, 21, 63-83.

Panjer, H.H. and Willmot, G.E., 1992. Insurance Risk Models. The Society of Actuaries.

Tsitsiashvili, G.Sh. and Konstantinides, D.G., 2001. Supertails in risk theory. Far Eastern Mathem. J., 2, 68-76. 